\title{
Transmission spectroscopy of the inflated exo-Saturn HAT-P-19b ${ }^{\star}, \star \star$
}

\author{
M. Mallonn ${ }^{1}$, C. von Essen ${ }^{2}$, J. Weingrill ${ }^{1}$, K. G. Strassmeier ${ }^{1}$, I. Ribas ${ }^{3}$, T. A. Carroll ${ }^{1}$, E. Herrero ${ }^{3}$, T. Granzer ${ }^{1}$, \\ A. Claret ${ }^{4}$, and A. Schwope ${ }^{1}$ \\ ${ }^{1}$ Leibniz-Institut für Astrophysik Potsdam, An der Sternwarte 16, 14482 Potsdam, Germany \\ e-mail: mmallonn@aip.de \\ 2 Stellar Astrophysics Centre, Ny Munkegade 120, 8000 Aarhus, Denmark \\ ${ }^{3}$ Institut de Ciències de l'Espai (CSIC-IEEC), Campus UAB, Facultat de Cències, Torre C5 parell, 2a pl, 08193 Bellaterra, Spain \\ ${ }^{4}$ Instituto de Astrofísica de Andalucía, CSIC, Apartado 3004, 18080 Granada, Spain
}

Received 7 March 2014 / Accepted 27 May 2015

\begin{abstract}
Context. Transiting highly inflated giant planets offer the possibility of characterizing their atmospheres. A fraction of the starlight passes through the high-altitude layers of the planetary atmosphere during transit. The resulting absorption is expected to be wavelength dependent for cloud-free atmospheres with an amplitude of up to $10^{-3}$ of the stellar flux, while a high-altitude cloud deck would cause a gray opacity.

Aims. We observed the Saturn-mass and Jupiter-sized exoplanet HAT-P-19b to refine its transit parameters and ephemeris as well as to shed first light on its transmission spectrum. We monitored the host star over one year to quantify its flux variability and to correct the transmission spectrum for a slope caused by starspots.

Methods. A transit of HAT-P-19b was observed spectroscopically with OSIRIS at the Gran Telescopio Canarias in January 2012. The spectra of the target and the comparison star covered the wavelength range from 5600 to $7600 \AA$. One high-precision differential light curve was created by integrating the entire spectral flux. This white-light curve was used to derive absolute transit parameters. Furthermore, a set of light curves over wavelength was formed by a flux integration in 41 wavelength channels of $50 \AA$ width. We analyzed these spectral light curves for chromatic variations of transit depth.

Results. The transit fit of the combined white-light curve yields a refined value of the planet-to-star radius ratio of $0.1390 \pm 0.0012$ and an inclination of $88.89 \pm 0.32 \mathrm{deg}$. After a re-analysis of published data, we refine the orbital period to $4.0087844 \pm 0.0000015$ days. We obtain a flat transmission spectrum without significant additional absorption at any wavelength or any slope. However, our accuracy is not sufficient to significantly rule out the presence of a pressure-broadened sodium feature. Our photometric monitoring campaign allowed for an estimate of the stellar rotation period of $35.5 \pm 2.5$ days and an improved age estimate of $5.5_{-13}^{+1.8}$ Gyr by gyrochronology. The calculated correction of the transit depth for unocculted spots on the visible hemisphere was found to be well within the derived $1 \sigma$ uncertainty of the white-light curve and the spectral data points of the transmission spectrum.
\end{abstract}

Key words. planetary systems - planets and satellites: atmospheres - starspots - stars: individual: HAT-P-19 techniques: spectroscopic

\section{Introduction}

Transiting extrasolar planets offer the opportunity of characterizing their atmospheres. During a planetary transit, parts of the starlight pass through an atmospheric ring at the planet's terminator region, picking up the signature of the planetary atmosphere. These spectral signatures are measurable with the current generation of instruments for some favorable planetary systems. The required spectroscopic precision necessary for the detection of these features depends on the planet-to-star radius ratio, the scale height of the planetary atmosphere, and the number of photons that can be collected from the host star

\footnotetext{
* Based on observations made with the Gran Telescopio Canarias (GTC), installed in the Spanish Observatorio del Roque de los Muchachos of the Instituto de Astrofisica de Canarias, in the island of La Palma, as well as on data obtained with the STELLA robotic telescope in Tenerife, an AIP facility jointly operated by AIP and IAC.

$\star \star$ Tables 1 and 3 are available in electronic form at

http://www . aanda.org
}

during transit. First attempts to detect additional absorption during transit were already able to provide upper limits (Rauer et al. 2000; Bundy \& Marcy 2000; Moutou et al. 2001). The sensitivity neccessary for a detection of an atmospheric signal was achieved for the first time by Charbonneau et al. (2002), who detected absorption due to sodium in the atmosphere of HD 209458b using HST/STIS optical observations. Today, transmission spectroscopy mainly comprises two observational approaches: medium to high-resolution spectroscopy (5000 $\lesssim \lesssim 100000$ ), and low-resolution spectrophotometry $(R \lesssim 200)$. The former one has a much higher sensitivity in detecting and resolving very narrow spectral features, whereas the latter technique is able to also reveal overall trends in the planetary spectrum that are caused by Rayleigh scattering, for example (e.g., Pont et al. 2008, 2013).

Most of the exoplanet targets for which the current generation of instruments is sensitive enough to characterize the atmosphere are close-in gas giants. For an Earth-like extrasolar planet the atmospheric ring is too thin to detectably modify the host 
star's light. The close-in gas giants, known as hot Jupiters, tend to have equilibrium temperatures ${ }^{1}$ of $1000 \mathrm{~K}$ and more. Their optical spectra might be dominated by broad atomic alkali absorption lines or, when hotter than about $1500 \mathrm{~K}$, by absorption of $\mathrm{TiO}$ according to theoretical investigations (Hubeny et al. 2003; Fortney et al. 2006, 2010). Clouds of condensed dust particles are another plausible scenario for hot Jupiters (Woitke \& Helling 2003; Helling et al. 2008) in similarity to L-dwarfs, which are often described by assuming a cloud layer. Fortney (2005) predicted that condensates even in very low densities in the probable atmosphere would significantly change the spectral appearance because of the slanted geometry under which the starlight passes through the planetary atmosphere.

Optical characterizations of hot Jupiters using spectrophotometry can already be found in the literature for multiple targets. These studies show a high diversity in their optical lowresolution spectral appearance.

There are robust detections of optical alkali absorption features resolved in low spectral resolution $R \leq 200$ for HD 209458b (Sing et al. 2008b), HAT-P-1b (Nikolov et al. 2014), XO-2 (Sing et al. 2012), and WASP-31b (Sing et al. 2015). Murgas et al. (2014) presented a probable detection of $\mathrm{Na}$ for WASP-43b and a tentative detection of $\mathrm{K}$ was given by Nikolov et al. (2015). The (low-resolution) non-detection in the atmospheres of HD 189733b and WASP-12b can be explained by scattering particles blocking the atmospheric heights that are possible for atomic sodium and potassium (Pont et al. 2008; Sing et al. 2013). The non-detection of alkali absorption in WASP-29b and HAT-P-32b can either be explained by clouds that strongly mute the spectral features or by subsolar abundances of sodium and potassium (Gibson et al. 2013a,b). Sing et al. (2011a) found significant $\mathrm{K}$ absorption in the atmosphere of $\mathrm{XO}-2 \mathrm{~b}$ using spectrophotometry with narrow-band tunable filters, giving a higher spectral resolution of $R \sim 800$.

A spectral slope in the optical that is indicative of Rayleigh scattering by aerosols has been found for HD 189733b (Pont et al. 2008, 2013), WASP-12b (Sing et al. 2013), WASP-6b (Jordán et al. 2013), and WASP-31b (Sing et al. 2015). In contrast, the optical spectra of HD 209458b and XO-2b are best explained by a clear atmosphere model (Sing et al. 2008b, 2011a, 2012). HD 209458b also features a Rayleigh slope shortward of $5000 \AA$, which is interpreted as scattering by $\mathrm{H}_{2}$ molecules instead of aerosols (Lecavelier Des Etangs et al. 2008b).

Indications for the presence of $\mathrm{TiO}$ in the terminator region was so far only found for HD 29458b (Désert et al. 2008) and WASP-12b; in the latter case, TiH is an alternative solution for the measurements (Stevenson et al. 2014). While this molecular absorption in HD 29458b still lacks an independent confirmation, a follow-up HST observation of WASP-12 found no longer any indication for TiO (Sing et al. 2013). Nor was TiO was detected in the probably cloud-free atmospheres of WASP-19b (Huitson et al. 2013) and XO-2b (Sing et al. 2011a). Furthermore, it was ruled out for the somewhat cooler atmospheres of HAT-P-1b (Nikolov et al. 2014). The non-detection of TiO in HD 189733b, HAT-P-32b, WASP-31b, and WASP-6b can be explained by clouds or haze on top of the atmosphere (Pont et al. 2008; Gibson et al. 2013b; Sing et al. 2015; Nikolov et al. 2015).

\footnotetext{
1 The equilibrium temperature is a theoretical temperature of the planetary atmosphere assuming a radiative balance between the integrated absorbed flux received from the star and the integrated emitted flux by the planet.
}

Here we report a search for sodium absorption in the atmosphere of HAT-P-19b using ground-based differential spectrophotometry. This ground-based technique was pioneered by Bean et al. (2010) for the super-Earth GJ1214b as the search for chromatic transit depth variations among a set of simultaneously observed light curves. We observed one transit event with the optical spectrograph OSIRIS at the Gran Telescopio de Canarias. Several studies already proved the capability of OSIRIS for optical transit spectrophotometry (Sing et al. 2011a, 2012; Colón et al. 2012; Murgas et al. 2014).

HAT-P-19b is a hot Jupiter with a period very close to four days (Hartman et al. 2011, hereafter H11). It is similar to Jupiter in size $\left(1.13 R_{\mathrm{J}}\right)$ and to Saturn in mass $\left(0.29 M_{\mathrm{J}}\right)$, giving it a very low surface gravity of about $6 \mathrm{~m} / \mathrm{s}^{2}$. The equilibrium temperature was estimated by $\mathrm{H} 11$ to be $1010 \mathrm{~K}$. Hence, the scale height is $\sim 600 \mathrm{~km}$ assuming Jupiter's mean molecular weight of 2.2 $\mathrm{g} /$ mole. The planet transits a K-type main sequence star of $V=$ $12.9 \mathrm{mag}, M=0.84 M_{\odot}$ and $R=0.82 R_{\odot}$. A great advantage for differential spectrophotometry is the presence of a near-by reference star, very similar in brightness, just 1.5 arcmin away and of similar spectral type.

If present, brightness inhomogeneities on the stellar surface affect the derivation of transit parameters and mimic a slope in the transmission spectrum. The knowledge of a long-term flux variation and the stellar flux level at the time of the transit observation can be used to correct for this influence. For this reason, we performed a two-color monitoring campaign for HAT-P-19 using the $1.2 \mathrm{~m}$ telescope STELLA on Tenerife over a time span of about 300 days. Similar studies can be found in the literature for the hot-Jupiter host stars HD 189733 (Pont et al. 2013), WASP-19 (Huitson et al. 2013), WASP-12 (Sing et al. 2013), HAT-P-1 (Nikolov et al. 2014), WASP-6 (Nikolov et al. 2015), and WASP-31 (Sing et al. 2015).

This paper is structured as follows: Sect. 2 describes the observations and the data reduction, and Sect. 3 presents the analysis and results. A discussion of the results is given in Sect. 4, followed by the conclusions in Sect. 5 .

\section{Observations and data reduction}

We observed the exoplanet host star HAT-P-19 in service mode during one transit event with the Gran Telescopio Canarias (GTC) located at the Observatorio del Roque de los Muchachos on the island of La Palma and operated by the Instituto de Astrofísica de Canarias. We monitored the star with the STELLA $1.2 \mathrm{~m}$ telescope of the Leibniz-Institute for Astrophysics Potsdam (AIP) installed in the Observatorio del Teide on the island of Tenerife.

\subsection{Spectroscopic GTC OSIRIS observation}

\subsubsection{Instrument setup}

During the transit observation we made use of GTC's optical spectrograph OSIRIS. The dispersive element was the VPH R2500R, providing a wavelength coverage of 5600 to $7500 \AA$ and a dispersion of about $1 \AA$ per binned pixel. We used the widest long slit available at the time of the observation, the 5.0" slit, which caused the spectral resolution to be seeing limited to roughly $R \sim 1000$. We read out the two Marconi CCD detectors in the fastest available, $500 \mathrm{kHz}$ read-out full-frame mode. The standard $2 \times 2$ binning resulted in less than $10 \mathrm{~s}$ closed-shutter time between the exposures. Exposure time was $60 \mathrm{~s}$. 
We chose a rotation angle of the instrument that centered (in dispersion direction) both the target HAT-P-19 and the reference star GSC 0228301197 (USNO-B1 1246-0009792) in the slit for simultaneous observations. OSIRIS covers its field of view with two CCD chips; we placed both objects on chip 1 to avoid potential differences between chip 1 and chip 2 as error sources in the differential spectrophotometry.

\subsubsection{Observing log}

The transit observation was conducted on January 10, 2012. The target was observed for slightly less than five hours from 19:33 to 00:19 UT, resulting in 238 exposures. We typically reached a signal-to-noise ratio $S / N \sim 380$ per pixel in dispersion at central wavelengths. The transit lasted from 19:56 to 22:39 UT (first to fourth contact). The night was photometric with the seeing varying from $0.9^{\prime \prime}$ to $1.3^{\prime \prime}$. Nevertheless, the observing conditions were far from optimal for high-precision spectrophotometry. The observation began shortly after the meridian passage of HAT-P19 , which occurred very close to the zenith. Unfortunately, the dome construction of the GTC could not be fully opened at the time of the observation, causing vignetting at the highest elevations. This vignetting was different for target and comparison star, clearly distracting the transit light-curve (Fig. 3) during pretransit phase. Moreover, the target set quickly during the time series to a maximum airmass of 3.23 , leading to a rapid spread of the point spread function with time. In Fig. 1 the evolution of the flux level, airmass, full width at half maximum (FWHM), and detector position relative to its mean is shown. The point spread function became asymmetric at about half the observing time due to a loss of focus; the observations were not stopped for focus adjustment to avoid further systematics introduced by an abrupt change of the telescope settings. Therefore the obtained values for the FWHM and pixel position need to be treated with caution because they were derived by fitting a Gaussian function to the spatial spectral profile. However, the guiding system kept the spectrum almost at the same position during the entire time series, it moved in spatial direction by less than 1 pixel. Two jumps in pixel position occurred, which were of subpixel value and had no effect on the resulting light curves.

\subsubsection{Data reduction}

The OSIRIS data were reduced by routines written in ESOMidas. Standard calibration frames have been obtained at the same day as the science observations. Flatfield frames were exposed through the $5^{\prime \prime}$ wide long slit, the same slit as used for the science frames. However, calibration spectra of $\mathrm{HgAr}$ and Ne lambs were taken with a $1^{\prime \prime}$ wide slit to avoid unpractically broad emission lines. A bias subtraction of the flat, arc lamp, and science frames was performed using the overscan regions. The flatfield correction was done by a master flat created of the fluxweighted average of 101 single flatfield frames taken the same day. We noticed that the flatfield correction had almost no effect on the quality in terms of scatter on the final transit light-curves or on the absolute value of the transit parameters. For example, the difference in the planet-to-star radius ratio $k$ whether a flat field correction was applied or not was about ten percent of the derived $1 \sigma$ uncertainty.

Before the wavelength calibration we corrected for a drift of the spectra in dispersion direction of about 1.4 pixel over the length of the time series. The origin of this drift is most probably a combination of telescope flexure, obvious in a shift of the skylines by a total of about 0.4 pixel, and a drift of the chromatic centroids of the objects in the long slit, probably caused by field rotation residuals and differential atmospheric refraction (OSIRIS does not include an atmospheric dispersion corrector). For simplicity we corrected for the drift achromatically. A difference in drift among the two objects has just been found for very high airmass, data that were excluded in the later analysis (see Sect. 3.1), hence we applied the same drift correction over the entire spatial range. In the through-slit acquisition image, taken in the Sloan $r$ band, we measured a slight mismatch of the object centroids in dispersion of $\sim 0.4$ pixel $\left(\sim 0.1^{\prime \prime}\right)$, which we also corrected for after the wavelength calibration and spectral extraction. In summary, we estimate the wavelength calibration to be accurate to just about $0.5 \AA$ due to residual wavelength dependent shifts and stresses over time both in dispersion and spatial direction.

In the presence of curved sky emission lines, we decided for a two-dimensional wavelength calibration of the whole frame to allow for sky estimation in spatially extended stripes distant to the spatial center of the object. While the choice of the sky stripe width minimized the light-curve scatter by up to ten percent depending on the wavelength region, it influenced the absolute transit parameters always by less than their $1 \sigma$ error values and did not affect the derived transmission spectrum either. The final width of the sky stripes spatially above and below the spectrum was 100 pixel each. The stripes were separated from the object extraction stripe by a 50 pixel gap due to the very extended wings of the spectral profile. The sky value at the object position was estimated by a linear interpolation between these two sky stripes, independently derived for each pixel in dispersion direction.

The widths of the aperture in which to extract the object flux were chosen to scale with the spatial FWHM of the spectral profile to account for the immense expansion of the point spread function at higher airmass (Fig. 1). A set of scaling factors were tested to minimize the scatter in the white-light curve. The final aperture size was 7.5 times the FWHM, which ranges from 25 to 105 pixel within the time series. Different widths of the sky stripes were alos tested. The scatter of the white-light curve decreased slowly with increasing width until it reached a plateau at about 100 pixels. We determined whether the optimal extraction technique of Horne (1986) could further minimize the dispersion on the light curves, but as expected for very high $\mathrm{S} / \mathrm{N}$ spectra, there was no significant difference to a simple flux sum in spatial direction. Instead, the simple flux sum yielded more robust results in the few regions with hot or dark pixels. The onedimensional spectra were extracted in pieces of steps $10 \AA$ wide to account for the wavelength-dependent FWHM of the spectral profile. Then the pieces were stitched together in wavelength, forming spectra from 5617 to $7687 \AA$. The spectra of exposure 1 are shown in Fig. 2.

The spectra were then divided into wavelength channels of a certain width (see Fig. 2 and Sect. 3.3). For each channel the flux of both objects, target and comparison star, was integrated and used to perform differential photometry. In this way, a set of simultaneously observed light curves consecutive in wavelength was achieved. Individual photometric errors were estimated by

$\Delta F=\frac{\sqrt{A \sigma^{2}+\frac{F}{g}}}{F}$,

with $A$ as the area (in pixel) over which the total flux $F$ (in ADU) was summed, $\sigma$ the standard deviation of noise (in ADU) estimated in the two sky stripe areas, and $g$ the detector gain of 


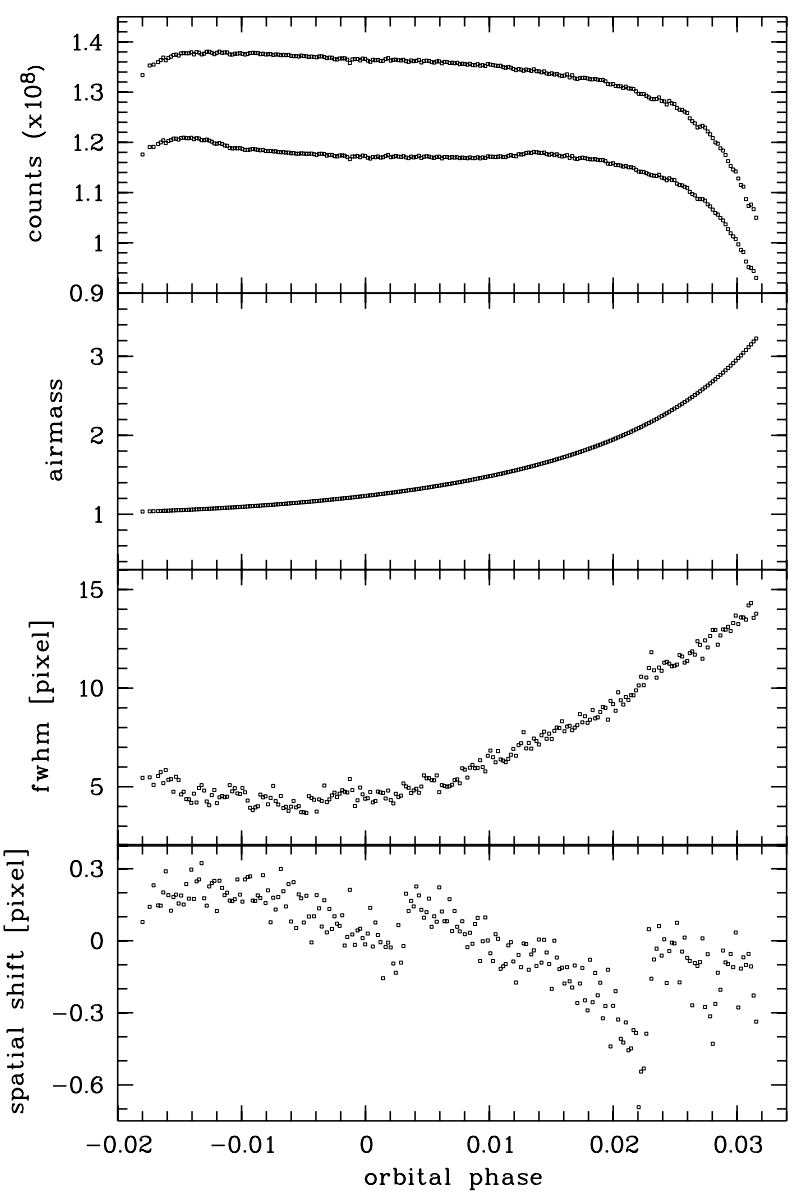

Fig. 1. Evolution of observational parameters over the time series. From top to bottom: count rate of target (lower flux level) and reference star (higher flux level) in ADU integrated over the spectral range, airmass, FWHM in spatial direction at central wavelength in pixel, and spectrum displacement in spatial direction in pixel.

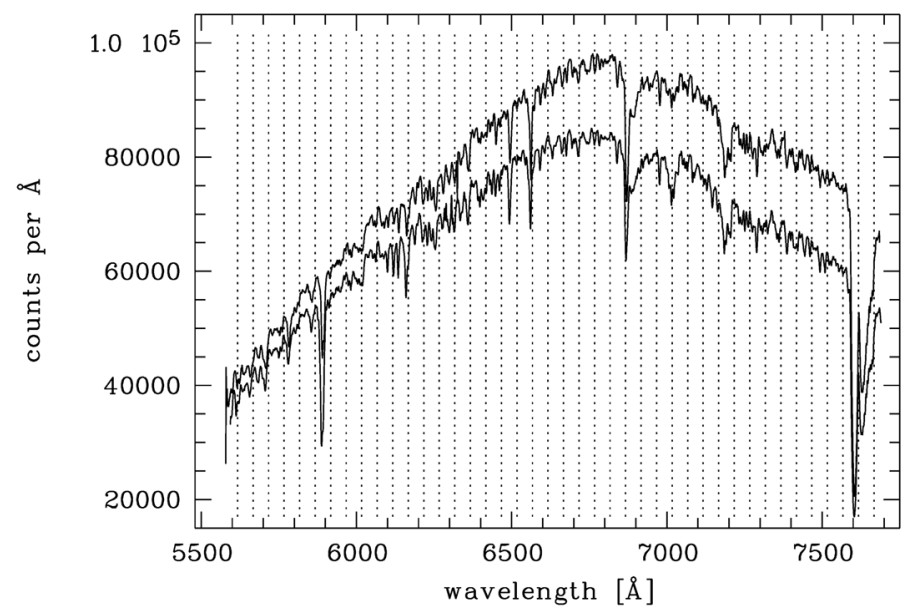

Fig. 2. Spectra of HAT-P-19 (lower flux level) and the comparison star (higher flux level) of the first exposure. The $50 \AA$ wavelength channels used for spectrophotometry are indicated by vertical dotted lines.

$g=1.46 \mathrm{e}^{-}$/ADU. This equation follows the photometric error estimation of the software package Source Extractor ${ }^{2}$ (Bertin \& Arnouts 1996).

\footnotetext{
2 www.astromatic.net/software/sextractor
}

\subsection{Photometric STELLA-WIFSIP monitoring}

\subsubsection{Instrument setup}

We observed HAT-P-19 with the wide-field imager WIFSIP of the robotic $1.2 \mathrm{~m}$ twin-telescope STELLA on Tenerife (Strassmeier et al. 2004; Weber et al. 2012). WIFSIP consists of a $4 \mathrm{k} \times 4 \mathrm{k}$ back-illuminated CCD with a plate scale of $0.322^{\prime \prime} /$ pixel and four read out amplifiers. It covers a field of 22 by 22 arcmin on the sky. The automatic scheduler of STELLA was set to observe HAT-P-19 on average every second night in blocks of three exposures in $V$ and three exposures in $I$ of $20 \mathrm{~s}$ exposure time each. We obtained 324 frames within 35 different observing nights from December 2011 to February 2012; between May 2012 and October 2012, the dataset contains 1268 frames from 82 nights.

\subsubsection{Data reduction}

The bias and flatfield correction was made with the STELLA data reduction pipeline, for details see Granzer et al. (in prep.). The following reduction steps were made with routines written in ESO-MIDAS. We conducted aperture photometry with the software package Source Extractor. Its MAG_AUTO option calculates an elliptic aperture individually for each image and object according to the second-order moments of the object's light distribution. This method provides the flexibility to account for the varying observing conditions over the ten-month observing time. The $I$-band data suffered from fringing, whose pattern was found to depend mainly on CCD detector temperature. We created master fringe maps by averaging the individual objectremoved and smoothed science frames in groups of the same CCD temperature. The fringe correction was made by subtracting the temperature-selected master fringe map, scaled in amplitude to match the fringes of the individual science frames. The fringe residuals typically had a strength of about 20 percent of the original fringe pattern.

We used the method of optimal weighting of an ensemble of comparison stars (Broeg et al. 2005) to form an artificial reference for differential photometry. We experimented with many different comparison star ensembles to verify that the observed photometric signal was independent of the choice of the comparison stars. The final ensemble is formed by three stars similar in brightness and color to HAT-P-19, read out through the same amplifier as the target. The same ensemble was used for the $V$ - and the $I$-band data. After averaging the three consecutively taken data points and subtracting a transit model using the transit parameter derived in Sects. 3.1 and 3.2, the point-to-point scatter of the data versus a sine model (see Sect. 3.4) was about $2.5 \mathrm{mmag}$ in both filters.

\section{Analysis and results}

We fit Mandel \& Agol (2002) transit light-curve models to all transit light-curves. The main parameters of the fit are the inclination $i$, the transit midpoint $T_{0}$, the limb-darkening coefficients of a quadratic law $u_{\mathrm{A}}$ and $v_{\mathrm{A}}$, the scaled semimajor axis $a / R_{\mathrm{A}}$, and the ratio of the planetary and stellar radius $k=R_{b} / R_{\mathrm{A}}$, where $a$ is the semimajor axis of the planetary orbit and $R_{b}$ and $R_{\mathrm{A}}$ are the absolute planetary and stellar radii. The best-fitting parameters were determined by a least-squares Markov chain Monte Carlo (MCMC) approach that made use of PyAstronomy ${ }^{3}$.

3 https://github.com/sczesla/PyAstronomy 


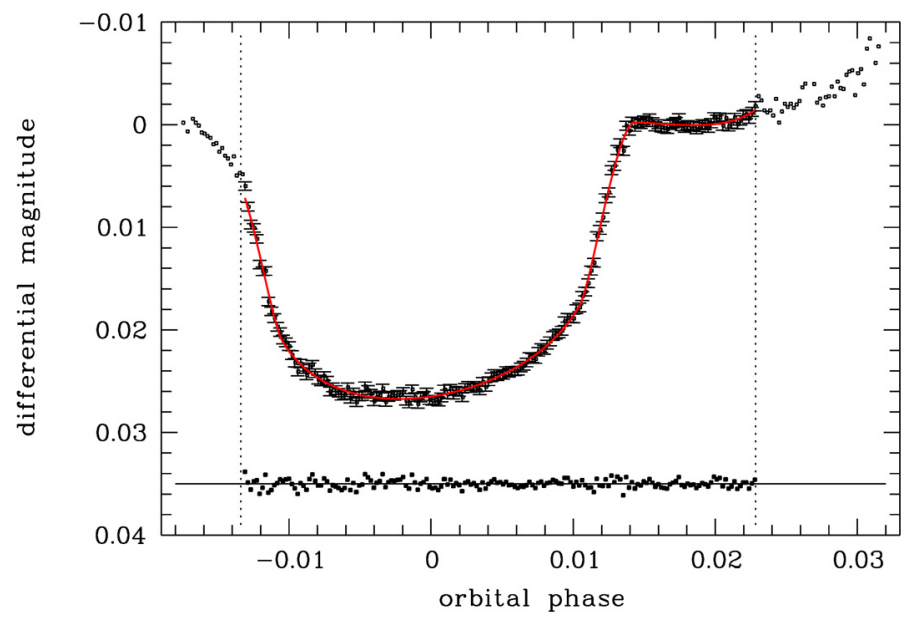

Fig. 3. Transit light-curve integrated over the entire spectral range (white light). The vertical dashed lines confine the data that were used for the analysis, see text for details. The red solid line shows the best-fit model, the residuals are presented at the bottom.

\subsection{Detrending and white-light curve analysis}

A white-light curve was formed by integrating the flux of the entire spectral range. As a very first step, we excluded the first 20 data points that lost flux because of the obscuration of the primary mirror by the dome construction. Unfortunately, these data formed our pre-ingress measurements. The resulting lightcurve features obviously smooth deviations from a theoretical symmetric transit light-curve, see Fig. 3. To model this deformation, we fitted a low-order polynomial to external parameters simultaneously to the transit model. Various polynomials to the external parameters time, airmass $z$, sky background, FWHM in spatial direction, pixel shift in $x$ (dispersion direction), and pixel shift in $y$ (spatial direction), and their combinations were tested. The pixel shift in spatial direction was measured by the fit of a Gaussian function to the spatial profile, the measurement of the pixel shift in dispersion was taken as the average of the centroid of six spectral features per object.

The fit parameters of the transit model were $a / R_{\mathrm{A}}, k, i$, and $T_{0}$ with the values determined by $\mathrm{H} 11$ as starting values plus their derived errors as Gaussian priors. The eccentricity and longitude of periastron were fixed to the values of $\mathrm{H} 11$. We ran ten chains of $1 \times 10^{5} \mathrm{MCMC}$ iterations, disgarded the initial $3 \times 10^{4}$ steps in every chain as burn-in phase, and merged all remaining iterations. The best-fit parameter values were derived as the mean of the posterior parameter distribution and the $1 \sigma$ uncertainties as the $68.3 \%$ of highest probability.

To avoid overfitting of the deformations andtrends in the light curve, we chose the function that minimized the Bayesian information criterion (BIC, Schwarz 1978). The BIC is similar to the goodness-of-fit estimation with a chi-square calculation, but adds a penalty term for the number of parameters in the model,

$\mathrm{BIC}=\sum \frac{(O-M)^{2}}{\sigma^{2}}+m \cdot \ln (n)$,

with $O$ in our case as the observation, $M$ as the transit and detrend model, $m$ the number of free parameters, $\sigma$ the photometric error of the individual data points, and $n$ the number of data points, here 175. We expect Eq. (1) to underestimate these values because of its ignorance of correlated noise. Therefore, we calculated a scaling factor to match the mean photometric error with the standard deviation of the photometric points.
To estimate this factor, we only used the post-transit data (phase $>0.015$ ) to avoid any bias introduced by a premature transit modeling. Then the BIC values for the tested low-order polynomials were calculated on the entire time series. Table 1 gives these values for a selection of tested detrending functions together with their derived transit parameters, which depend significantly on the detrending function choice. The final choice for the detrending function was a third-order polynomial with the airmass as independent variable because it minimized the BIC value, the scatter of the residuals (rms), and the amount of residual timecorrelated noise (estimated by the $\beta$ value, see below).

The stellar limb-darkening was accounted for by the quadratic law in the transit fit. The limb-darkening coefficients were spherically calculated for a star of $T_{\text {eff }}=5000 \mathrm{~K}$ and $\log g=4.5$ in high spectral resolution using PHOENIX stellar atmosphere models of metallicity $\log [\mathrm{M} / \mathrm{H}]=0.0$, see Claret et al. (2013) for details. The applied values for the white-light curve were found by a flux-weighted average of these nearly monochromatic values. To analyze the white-light curve, we fitted for the linear limb-darkening coefficient $u_{\mathrm{A}}$ and fixed the nonlinear term $v_{\mathrm{A}}$ to the theoretical value. The fitted value of the linear term agreed with the prediction to within about $1 \sigma$. This approach of fitting $u_{\mathrm{A}}$ but fixing $v_{\mathrm{A}}$ to its theoretical value allows for some flexibility to account for potential systematic differences between the star and its theoretical model spectrum used for determination of the limb-darkening parameters. Furthermore, it avoids the problem of enlarged uncertainties when fitting for both coefficients caused by the strong correlation between $u_{\mathrm{A}}$ and $v_{\mathrm{A}}$ (Southworth et al. 2007a; Southworth 2008; Johnson et al. 2008).

We briefly comment on the systematic error introduced by the metallicity difference between the PHOENIX models (solar metallicity) and HAT-P-19: H11 derived a value of $\log [\mathrm{M} / \mathrm{H}]=0.23 \pm 0.08$. We used the limb-darkening tables of Claret (2000) and Claret (2004) to estimate the systematic error to be on the order of 0.005 for the quadratic limb-darkening coefficient (the linear coefficient is a free parameter in our transit fit). The resulting systematic error on the derived transit parameters is much smaller than their uncertainties.

The individual photometric error bars were enlarged by a common factor to give a reduced chi-square value of unity, $\chi_{v}^{2}=1.0$, versus the fitted model. To account for correlated red noise, we derived in a second step an additional scaling factor as the ratio of the calculated and the theoretical standard deviation of the binned photometric residuals, often called the $\beta$ factor (Gillon et al. 2006; Winn et al. 2008). We binned the residuals in intervals from 10 to $20 \mathrm{~min}$ (about the duration of ingress) in one-minute steps (original time sampling is $\sim 1.1 \mathrm{~min}$ ), derived the factor in each case, and finally used their average as the $\beta$ factor to enlarge the individual photometric errors.

There are features in the noise in the post-transit light curve at phases later than about 0.023 visible to the eye that are believed to be systematics potentially caused by differential slit loss. For white noise alone, the uncertainty of the radius ratio $k$ should decrease approximately proportionally to the square root of the number of out-of-transit (OoT) data. Here it was worth trying whether the uncertainties actually decrease with fewer OoT data. And indeed, the uncertainty of the radius ratio $\Delta k$ reached a minimum at an OoT limit of phase 0.0228 . In addition, the uncertainties of the scaled semimajor axis $\Delta\left(a / R_{\mathrm{A}}\right)$, the inclination $\Delta i$, and the transit mid-time $\Delta T_{0}$ reached their minima at about this number of OoT data, therefore we discarded all later phases from the analysis. 
Table 2. Transit fit parameter of the white-light curve.

\begin{tabular}{ll}
\hline \hline Parameter & Value \\
\hline$a / R_{\mathrm{A}}$ & $12.37 \pm 0.21$ \\
$k$ & $0.1390 \pm 0.0012$ \\
$i$ [deg] & $88.89 \pm 0.32$ \\
$T_{0}-2400000$ [days] & $55937.38839 \pm 0.00011$ \\
$u_{\mathrm{A}}$ & $0.5736 \pm 0.0182$ \\
$v_{\mathrm{A}}($ fixed) & 0.1376 \\
$g_{b}\left[\mathrm{~ms}^{-2}\right]$ & $5.97 \pm 0.61$ \\
\hline
\end{tabular}

Table 4. Transit mid-times of HAT-P-19 and their residuals versus the ephemeris derived in this work.

\begin{tabular}{lrrl}
\hline \hline $\begin{array}{l}\text { Mid-time } T_{0} \\
(\mathrm{BJD}(\mathrm{TDB})-24000000)\end{array}$ & $\begin{array}{r}\text { Cycle } \\
\text { number }\end{array}$ & $\begin{array}{r}\text { Residual } \\
\text { (days) }\end{array}$ & Reference \\
\hline $55135.63128 \pm 0.00038$ & -193 & -0.00011 & Hartman et al. (2011) \\
$55167.70182 \pm 0.00040$ & -185 & 0.00014 & Hartman et al. (2011) \\
$55937.38829 \pm 0.00010$ & 7 & -0.00001 & This work \\
\hline $55496.41945 \pm 0.00075$ & -103 & -0.00255 & Naves, R. \\
$55524.48225 \pm 0.00062$ & -96 & -0.00124 & Muler, G. \\
$55528.49211 \pm 0.00057$ & -95 & -0.00017 & Ruiz, J. \\
$55885.27456 \pm 0.00050$ & -6 & 0.00045 & Ayiomamitis, A. \\
$55921.35111 \pm 0.00075$ & 3 & -0.00205 & Naves, R. \\
$56145.84362 \pm 0.00051$ & 59 & -0.00147 & Shadic, S. \\
$56173.90627 \pm 0.00066$ & 66 & -0.00031 & Garlitz, J. \\
$56270.11642 \pm 0.00047$ & 90 & -0.00099 & Zhang, L. \\
\hline
\end{tabular}

Notes. The upper part lists the values of the professional light curves that were used to derive the ephemeris. The lower part lists the values of the amateur light curves (ETD/TRESCA) that were tested in the analysis, but were not used in the end to derive the ephemeris.

The results of the white-light curve analysis roughly agree with the work of $\mathrm{H} 11$ and are given in Table 2 together with an estimate of the surface gravity of the planet $g_{b}$, calculated with Eq. (4) of Southworth et al. (2007b) using the value of the stellar velocity amplitude $K$ from H11.

\subsection{Ephemeris}

We used the white-light curve of our transit measurement and the publicly available two complete transits from $\mathrm{H} 11$, which were observed with the KeplerCam camera on the FLWO $1.2 \mathrm{~m}$ telescope, to recalculate the ephemeris. The FLWO transits were reanalyzed with our algorithms to guarantee consistency in the analysis. A first-order polynomial over time was used for detrending. The free transit parameter was the mid-time of the transit $T_{0}$. The parameters $a / R_{\mathrm{A}}, k$, and $i$ were fixed to the values derived from our white-light curve, given in Table 2. The quadratic limb-darkening coefficients were fixed to the values used by H11. The results of the mid-transit times are summarized in Table 4 and graphically presented in Fig. 4. We experimented with the coefficients, using the linear coefficient as free fit parameter or varying both in meaningful ranges. The tests showed very little effect of very few seconds on $T_{0}$, which is well inside the errors. H11 used the coordinated universal time (UTC) as time base for their barycentric Julian date (BJD) calculations. As recommended by Eastman et al. (2010), we converted all times to the barycentric dynamical time (TDB) using

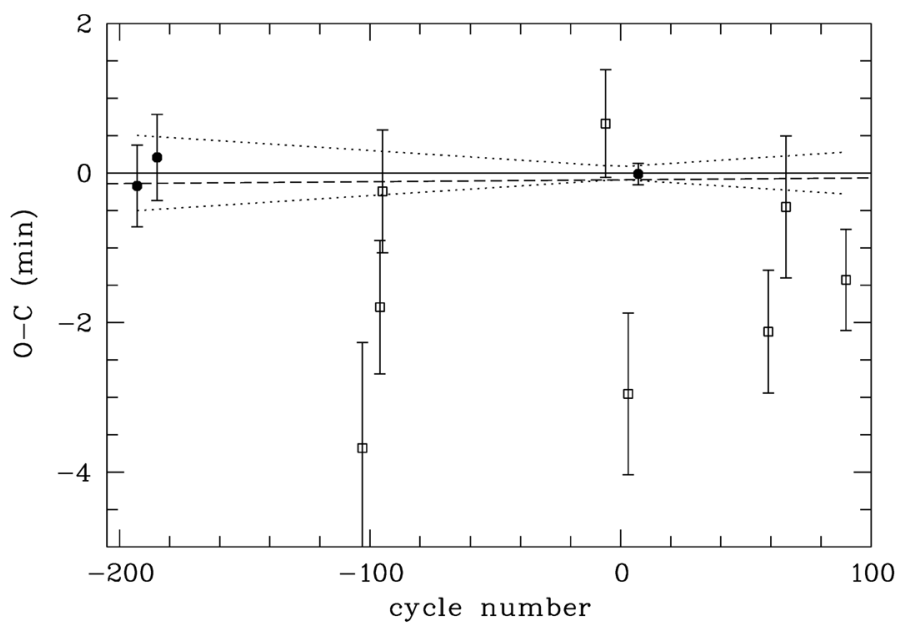

Fig. 4. Transit timing residuals versus the linear ephemeris of Eq. (3). The filled symbols represent the two transits of H11 and the transit of this work, which were used to derive the ephemeris. The open symbols show the amateur ETD transits. The dotted lines give the $1 \sigma$ uncertainty of the ephemeris as a function of cycle number. The dashed line shows the difference when the amateur data are included in the ephemeris fit, which yielded no significant improvement and was finally not used for Eq. (3).

their available online tools ${ }^{4}$. The newly found ephemeris is

$T_{\mathrm{c}}=\mathrm{BJD}(\mathrm{TDB}) 2455909.326808(55)+4.0087844(15) N$.

$T_{\mathrm{c}}$ is the predicted central time of a transit, $N$ is the cycle number with respect to the reference mid-time, and the numbers in brackets give the uncertainties of the last two digits. The reference mid-time was chosen to minimize the covariance between reference mid-time and period. The GTC transit accordingly corresponds to cycle 7 .

We tested whether additional transit light-curves observed by amateur astronomers could further improve the accuracy of the ephemeris. The data were made publicly available on the Exoplanet Transit Database (ETD) website ${ }^{5}$. We selected the ETD transits to have uncertainties on the mid-transit time $T_{0}$ of about one minute or less. The light curves were reanalyzed and the transit mid-times converted into BJD(TDB). However, we found that the time uncertainties of the eight ETD transits are too large to significantly improve the ephemeris of Eq. (3). Therefore we decided to use the better-documented and peerreviewed observations of $\mathrm{H} 11$ and our own observation. The timings of ETD roughly agree with Eq. (3), see Fig. 4, their residuals are all consistent with zero within less than $3 \sigma$.

\subsection{Transit depth as a function of wavelength}

We created two different chromatic sets of light curves by binning the flux of our spectra in wavelength channels of a certain width. At first, we chose $200 \AA$ as channel width, which yielded rather high $\mathrm{S} / \mathrm{N}$ per photometric data point, but a poor spectral resolution in our resulting transmission spectrum. Then we binned the flux in narrower channels, which provided a higher spectral resolution at the cost of noisier light curves and therefore higher uncertainties of the transit parameter. We performed a simple estimation of the channel width that was most sensitive to narrow spectral features with the help of a theoretical $1000 \mathrm{~K}$

\footnotetext{
4 http://astroutils.astronomy.ohio-state.edu/time/

5 http://var2.astro.cz/ETD/
} 


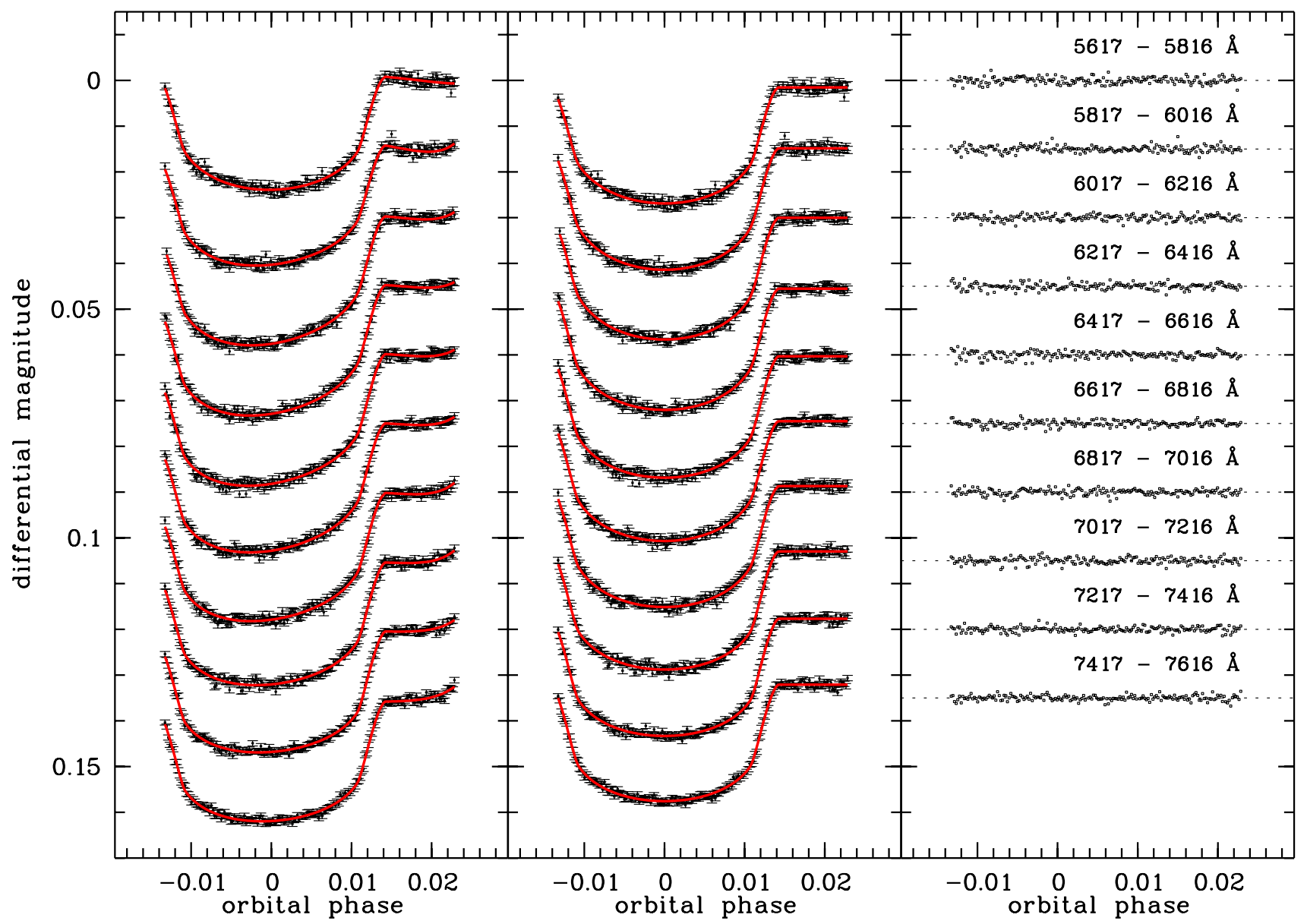

Fig. 5. Chromatic set of transit light-curves of HAT-P-19b, flux integration made in $200 \AA$ wide flux channels. Left panel: the raw light curves, middle panel: detrended light curves, right panel: the light-curve residuals.

transmission spectrum (Fortney et al. 2010). We compared the theoretically predicted transit depth in a channel centered on the $\mathrm{Na} \mathrm{D}$ line core with the transit depths in the adjacent channels for channel widths of 10 to $150 \AA$. The function of differential transit depths over channel width was weighted with a simple squareroot law to account for the $\mathrm{S} / \mathrm{N}$ dependence on the width. For simplicity we assumed here photon noise to dominate the noise budget. The resulting function peaks at about $50 \AA$ A. Figure 5 shows the light-curve set of $200 \AA$ channel width. In the left column the raw light curves are shown, in the middle column the detrended light curves, and in the column to the right the residuals versus the detrend + transit model.

The chromatic set of light curves was analyzed simultaneously. The fitting parameters per light curve were the four coefficients of the airmass-dependent third-order polynomial, $u_{\mathrm{A}}$, and $k$. In addition, we fitted for a common value per set of $a / R_{\mathrm{A}}$, $i$, and $T_{0}$ with the white-light curve uncertainties of Table 2 as Gaussian priors. In total, the number of simultaneously fitted parameters were 63 for the 10 light curves of $200 \AA$ width, and 249 for the 41 light curves of $50 \AA$ width. The results are presented in Table 3.

We verified the MCMC results of $k$ over wavelength with another available transit-modeling package, JKTEBOP 6 (Southworth et al. 2004). It uses the Levenberg-Marquardt optimization algorithm to find the best-fitting model and includes multiple options to give reliable error estimations. Here, we

\footnotetext{
6 www.astro.keele.ac.uk/jkt/codes/jktebop.html
}

calculated the errors with a Monte Carlo simulation (Southworth et al. 2005) and a residual-permutation algorithm (Jenkins et al. 2002; Southworth 2008), the latter being sensitive to correlated noise. We adopted the higher value of both. The vast majority of best-fit parameters agrees within one sigma, and all conclusions derived in this work about the measured transmission spectrum of HAT-P-19b are the same for both the MCMC analysis and JKTEBOP analysis. On average, the uncertainties of JKTEBOP were larger by about $20 \%$ than the uncertainties of the MCMC chains. This deviation does not change the results of this work, and a detailed analysis of its origin is beyond the scope of this work.

The transmission spectrum plotted in Fig. 6 appears very flat without significant outliers. We calculated the $\chi^{2}$ of the $50 \AA$ data set versus a Rayleigh-scattering slope given by $\mathrm{d} R_{b} / \mathrm{d} \ln \lambda=-4 H$ similar to the observed spectrum of HD 189733b (Lecavelier Des Etangs et al. 2008a) with $H$ as the atmospheric scale height. We also compared the data to a 1D solar metallicity model using a planet-wide averaged P-T profile computed by Fortney et al. (2010) for the parameters of the HAT-P-19 system, see Fig. 9. Both models were binned in the same $50 \AA$ wavelength channels as the observed spectra. The cloud-free model shows a prominent sodium absorption feature with pressure-broadened wings, also predicted by other modeling work for HAT-P-19b's equilibrium temperature of about 1000 K (Seager \& Sasselov 2000; Brown 2001; Hubbard et al. 2001). A similar feature of potassium causes the upward slope of planetary size in the model at the red end of our wavelength 


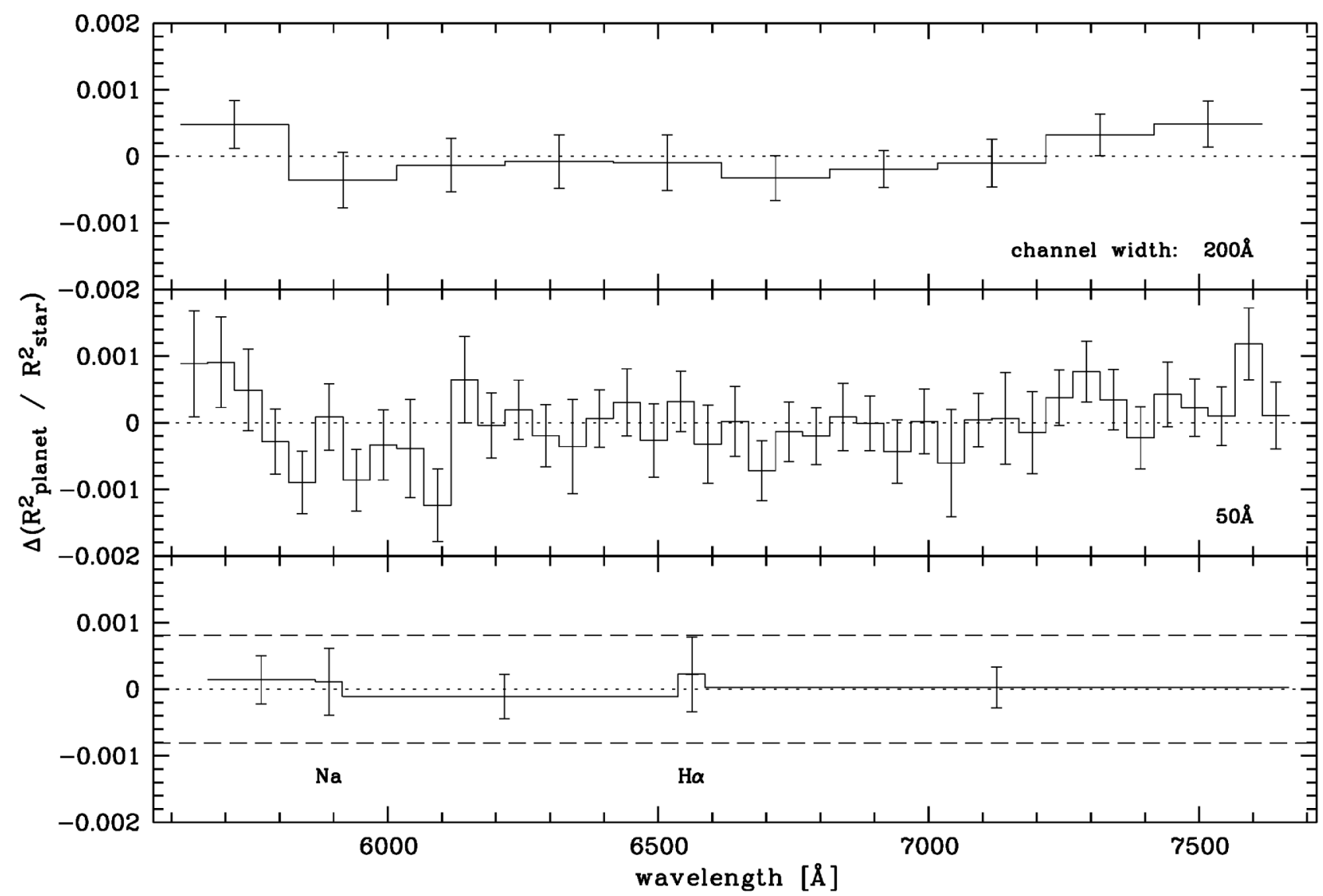

Fig. 6. Transmission spectrum of HAT-P-19b. In the upper panel the relative change in transit depth is shown for $200 \AA$ wide wavelength channels, in the middle panel a channel width of $50 \AA$ is presented. For the lower panel two $50 \AA$ wide channels concentrated on the line center of the Na doublet at $\sim 5892 \AA$ and $\mathrm{H} \alpha$ at $\sim 6563 \AA$. No significant variation was detected. The horizontal dotted line in the lower panel marks the average value, the dashed lines denote the difference corresponding to three atmospheric scale heights.

range, the line core of potassium was not covered, unfortunately. The lowest $\chi^{2}$ of 34.7 for $40 \mathrm{deg}$ of freedom is given by a flat line, 36.8 was obtained for the Rayleigh-scattering spectrum and 48.9 was the value for the cloud-free atmosphere.

We tried to test the influence of certain steps of our analysis on the obtained transmission spectrum. For example, we shifted the flux channels in wavelength by fractions of their width, we tested different lengths of post-transit baseline and the effect of fixing $a / R_{\mathrm{A}}$ and $i$ to the values found by H11. We also varied our treatment of the limb-darkening coefficients by fixing both $u_{\mathrm{A}}$ and $v_{\mathrm{A}}$ to theoretical values, another time we fitted for both in the transit modeling. No test yielded a significant deviation in spectrum shape from the spectrum presented here in Fig. 6.

\subsection{Photometric variability of HAT-P-19}

We found a clear periodic variation of HAT-P-19 in the longterm photometry shown in Fig. 7. A least-squares sine fit to the data revealed a period of $35.5 \pm 2.5$ days. The error value is drawn from the FWHM of the peak in the periodogram in Fig. 8. The amplitude was different for the used filters, $V$ and $I$, with $4.7 \pm 0.5$ and $3.0 \pm 0.4$ mmag provided by the least-squares sine fit. There are indications of varying period and amplitude in our data. When analyzing only the data of the observing season 2012, we obtain a stronger amplitude of $6.3 \pm 0.6$ and $4.6 \pm 0.5 \mathrm{mmag}$ for $V$ and $I$ and a slightly longer period of $38.6 \pm 4.4$ days. In the interpretation of the flux variation that is caused by starspots rotating in and out of view, these changes in period and amplitude are possibly related to spot evolution. However, the period variation is within one $\sigma$ and the amplitude variations do not exceed $3 \sigma$.

The periodogram in Fig. 8 shows three additional peaks next to the main signal at somewhat shorter period. They loose most of their power or disappear entirely when subtracting the most significant signal (pre-whitening). Instead, in the residual data we find another signal above the false-alarm probability of 0.01 at about 23 days. This period might also be related to spot evolution, but an in-depth period analysis is beyond scope of the current work.

H11 did not mention a stellar flux variability. We reanalyzed the HATnet photometry observed from September 2007 to February 2008 to search for the same periodicity. The available time series ${ }^{7}$ was treated by the external parameter decorrelation technique and the trend-filtering algorithm, as is common for HATnet data (see H11 and references therein). When we calculated a Lomb-Scargle periodogram, we found a significant period of $P=37.7 \pm 3.1$ days (false-alarm probability FAP $<$ 0.0002 ), see Fig. 8. The sine fit reveals a much lower variability amplitude than the STELLA data of only $1.0 \pm 0.3 \mathrm{mmag}$.

\subsection{Correction for starspots}

A likely reason for the periodic flux variation of HAT-P-19 are cool spots rotating in and out of view since the variation

7 http://www.hatnet.org/planets/discovery-hatlcs.html 


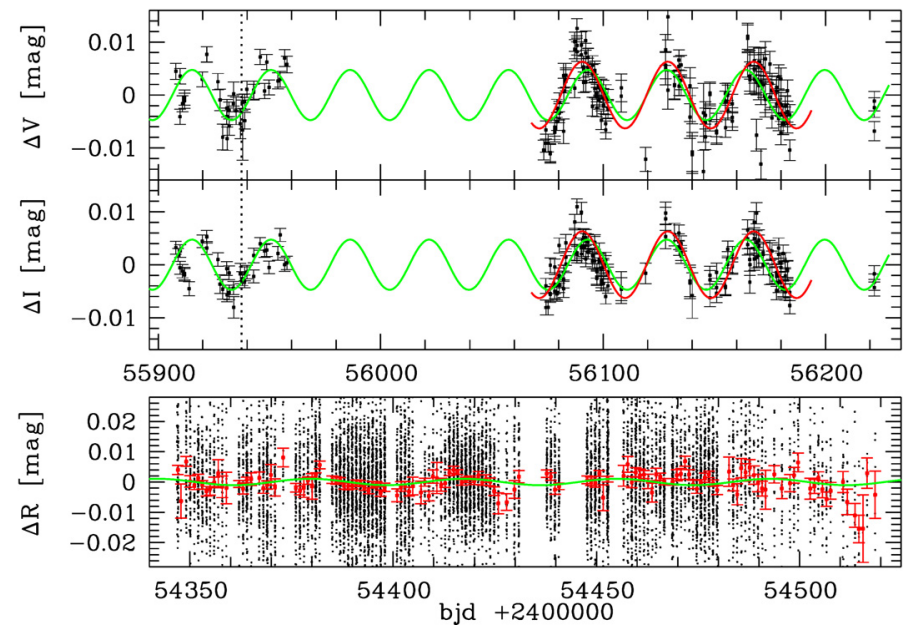

Fig. 7. Upper panel: STELLA differential $V$ band photometry. A simple sine model of the 2011+2012 data set overplotted in green, a sine model of just the 2012 data in red. The vertical dotted line marks the transit time of our spectroscopic observation. Middle panel: the same as in the upper panel, here for filter I. Lower panel: the HATnet differential $R$ band photometry from $2007 / 2008$. Black dots are the individual data points, red is the nightly average and green gives the best fit sine model.

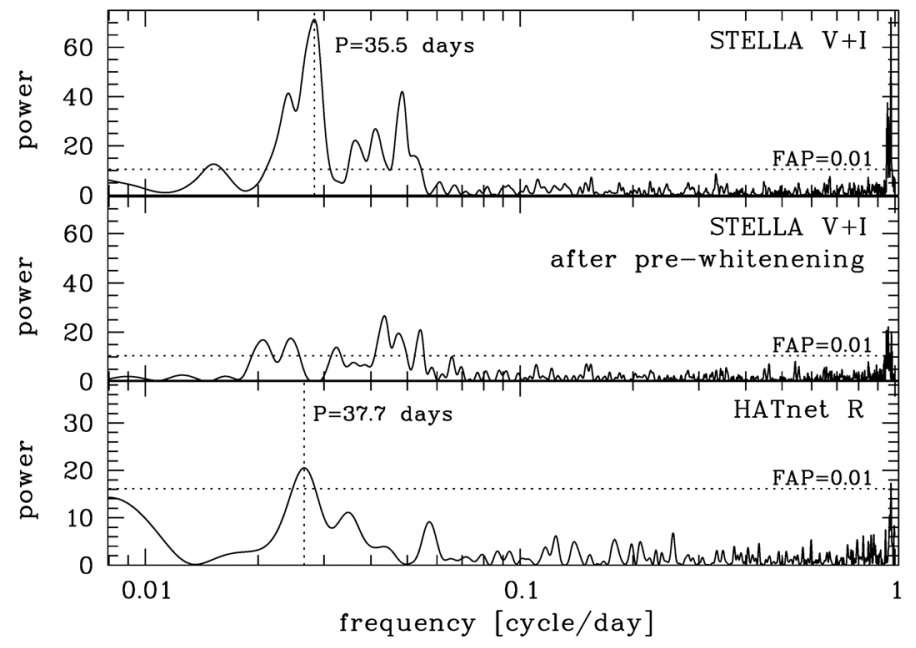

Fig. 8. Lomb-Scargle periodogram for the STELLA photometry (upper panel) and the HATnet photometry (lower panel). Horizontal dashed lines denote the power value of false-alarm probability (FAP) 0.01 . Vertical dashed lines mark the period found by the sine fit. Middle panel: STELLA periodogram after one pre-whitening step. Significant frequencies remain, which we attribute to the sampling pattern and/or spot evolution.

amplitude is larger in the $V$ band than in the redder $I$ band. Brightness inhomogeneities on the stellar surface influence the transit parameters (Pont et al. 2008; Czesla et al. 2009). An occultation of a starspot by the transiting planet causes a bump in the light curve, which leads to a shallower transit and an underestimation of the planet size if uncorrected. Starspots unocculted from the planet cause the average brightness to be higher along the transit chord than on the rest of the stellar hemisphere, which leads to deeper transits and an overestimation of the planet size. Examples of active host stars with frequently observed starspot crossings are HD 189733 (see Pont et al. 2013 and references therein) and WASP-19 (Tregloan-Reed et al. 2013; Huitson et al. 2013).
The white-light curve gives no evidence of a starspot crossing event. Possible scenarios that allow for spots along the chord without resolvable deformations of the transit shape such as very small spots or a homogeneous spot distribution along the chord are considered to either be negligible in their effect or unlikely. We therefore assume that the transit depth is not underestimated. With the long-term photometry on hand, we can estimate the influence of unocculted starspots on the stellar hemisphere visible at the moment of the transit event. This estimation depends on three quantities: The stellar flux level $f_{\text {meas }}$ at the time of the transit observation, the stellar flux level without spot dimming $f_{\text {quiet }}$, and the effective temperature of the spots $T_{\text {spot }}$. We define the relative variation in flux $\Delta f$ by

$\Delta f=\frac{f_{\text {meas }}-f_{\text {quiet }}}{f_{\text {quiet }}}$,

with $f_{\text {meas }}$ as the measured stellar flux and $f_{\text {quiet }}$ as the flux of the quiet star without brightness inhomogeneities. Désert et al. (2011) described how $\Delta f$ simply translates into the change in transit depth by

$\frac{k_{\text {meas }}^{2}-k_{\text {true }}^{2}}{k_{\text {true }}^{2}}=\alpha \Delta f$.

The proportionality factor $\alpha$ was estimated to be -3 in the system HD 189733 (Désert et al. 2011). Other studies simplified Eq. (5) for other planetary systems by $\alpha=-1$ (e.g., Berta et al. 2011). We cannot measure this on our own without transit measurements at multiple epochs, therefore we followed the simplest approach and set $\alpha$ to -1 .

A value of the flux variation $\Delta f$ includes the knowledge of HAT-P-19's flux level $f_{\text {quiet }}$ without any spots on its visible surface. Spots were probably also present at the time of the highest count rate of our light curve, therefore $f_{\text {quiet }}$ needed to be estimated. We approximated the level of a permanent flux dimming by the level of the variance of the long-term light curve, an approach undertaken by Pont et al. (2013) for HD 189733 based on the work of Aigrain et al. (2012). Thus, at the time of our transit observation we estimate $\Delta f \sim-0.007$ in $V$ band. Conservatively, we rounded this value to $1 \%$ flux dimming by spots.

To estimate $\Delta f$ over the spectral range covered by our transit observation, a value of the starspot temperature is needed. However, $T_{\text {spot }}$ cannot easily be derived by photometric data because of a degeneracy between spot size and spot temperature (Strassmeier 2009). In principle, this can be broken by multicolor photometry, but our error bars on the subpercent variation amplitudes in $V$ and $I$ band cannot reasonably constrain the temperature contrast between spots and photosphere. The starspots of the exoplanet host star HD 189733, similar in stellar parameters to HAT-P-19, have temperatures of between $750 \mathrm{~K}$ (Sing et al. 2011b) and $1000 \mathrm{~K}$ (Pont et al. 2008) lower than the spot-free photosphere. Berdyugina (2005) listed several early$\mathrm{K}$ dwarfs with spots of about $1500 \mathrm{~K}$ temperature contrast. We calculated spot corrections on the transmission spectrum for three different temperature contrasts, $500 \mathrm{~K}, 1000 \mathrm{~K}$, and $1500 \mathrm{~K}$. The spectral energy distributions of the spots and spotfree photosphere were approximated by blackbody radiation. Furthermore, we neglected the contribution of faculae. Under these assumptions, a flux dimming of one percent corresponds to about $2.3(1.4,1.1)$ percent coverage of the stellar hemisphere with spots of $500 \mathrm{~K}(1000 \mathrm{~K}, 1500 \mathrm{~K})$ temperature contrast. The obtained $\Delta k^{2}$ correction values over wavelength range from $\sim 1 \%$ 
of the transit depth $\left(\Delta k^{2} \sim 0.00019\right)$ at the blue end of our spectrum to about $0.8 \%\left(\Delta k^{2} \sim 0.00016\right)$ at the red end. The difference in $\Delta k^{2}$ according to the different spot temperatures is at the level of $0.1 \%$ of the transit depth at $7500 \AA$. Our derived uncertainty of the transit depth $k^{2}$ for the white-light curve is about $1.7 \%$. Hence, the spot correction on the absolute transit depth is of minor importance, and the correction on the relative changes of transit depth over wavelength is negligible.

\section{Discussion}

\subsection{Transmission spectrum}

We ran a simple calculation to test whether any potential variations in transit depth among the narrow wavelength channels might be blurred by the point spread function. We assumed the FWHM in dispersion direction to equal the measured FWHM in spatial direction due to the $5^{\prime \prime}$ wide slit. While the FWHM increased to more than ten pixel toward our OoT phase limit of 0.0228 , the average value during second and third contact of the transit was 4.8 pixel (1.2" on the sky). The corresponding light leakage weakens a potential difference in transit depth of the sodium $50 \AA$ channel with respect to the adjacent channels by $\sim 3 \%$. Hence, we neglect this effect here.

Key values for an initial characterization of an exoplanet atmosphere are the error bars of the relative transit depth measurements. We found the average error value to be higher than the scatter of the $k^{2}$ values for both the $50 \AA$ and the $200 \AA$ lightcurve sets. The reduced $\chi^{2}$ values of 0.9 and 0.7 , respectively, versus a constant $k^{2}$ confirm this indicator of a slight overestimation of the error bars. We compared our achieved $200 \AA$ accuracy to similar investigations present in the literature by a search for values of ground-based single-transit analyses using a comparable width of the flux channels in about the same wavelength range. We caution that differences can occur due to different telescope size, target brightness, and the complexity of systematics in the light curves and analysis techniques. However, our average uncertainty of $k^{2}$ for the $200 \AA$ channels of $3.6 \times 10^{-4}$ agrees well with the range of literature values $1.8-3.4 \times 10^{-4}$ (Gibson et al. 2013a,b; Jordán et al. 2013; Murgas et al. 2014). One reason for our uncertainty to be slightly larger is certainly the lack of an out-of-transit baseline on one side.

Our transmission spectrum of HAT-P-19b favors a featureless flat spectrum, but although less likely, the $\mathrm{Na}$ rich, cloud-free atmosphere overplotted in Fig. 9 is not ruled out. If confirmed by follow-up observations, a non-detection of the pressure-broadened $\mathrm{Na}$ absorption could be explained in two ways: first, a cloud or haze layer does not allow probing the deeper layers of the atmosphere and covers the broad alkali line wings. As a result of the slanted viewing geometry during transmission spectroscopy, even condensates of rather low abundance can make the atmosphere opaque (Fortney 2005). Observations of higher spectral resolution are needed to search for a spectroscopic feature at the line core. The other explanation would be a clear atmosphere with a depletion of atomic sodium. Sodium condensation into clouds of sodium sulfide, for instance, at the planets cooler night side could potentially decrease the amount of atomic sodium at the terminator region probed by our measurement (Lodders 1999). Another potential cause of atomic $\mathrm{Na}$ depletion is ionization by stellar UV photons (Fortney et al. 2003), a scenario which is plausible because we found HAT-P-19 to be magnetically active.

Among the known sample of hot-Jupiter exoplanets that have already been investigated by transmission spectroscopy there

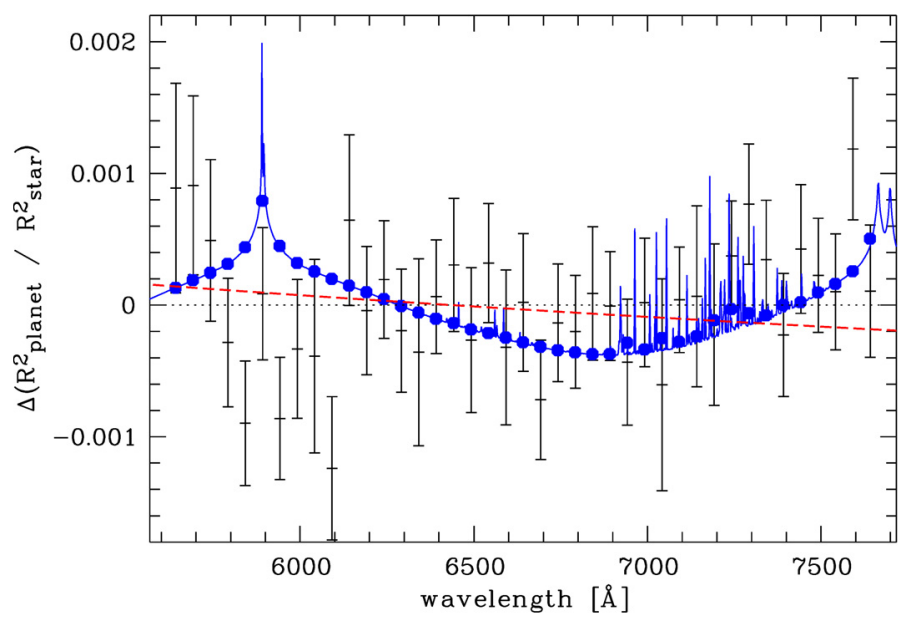

Fig. 9. Derived transmission spectrum in comparison to a theoretical spectrum calculated by Fortney et al. (2010) illustrated as a continuous blue line. The model values integrated over $50 \AA$ flux channel width are shown as blue filled circles. We also present a spectrum typical for Rayleigh-scattering as a red dashed line. Both models were computed according to the system parameters of HAT-P-19.

are three planets similar to HAT-P-19b in their system parameters: HAT-P-1b (Bakos et al. 2007), HAT-P-12b (Hartman et al. 2009), and WASP-29b (Hellier et al. 2010). All these planets have roughly the mass of Saturn, roughly the size of Jupiter, and an equilibrium temperature of 1000 to $1200 \mathrm{~K}$. They orbit solarlike main-sequence stars of spectral type G or K. Gibson et al. (2013a) obtained an optical transmission spectrum of WASP$29 \mathrm{~b}$ around the sodium line and was able to significantly rule out a pressure-broadened absorption feature. Nikolov et al. (2014) observed HAT-P-1b in the optical regime during transit and detected additional absorption at the sodium core in a $30 \AA$ wide flux channel, but no spectral signature of the broadened line wings. Line et al. (2013) observed HAT-P-12 in the near-IR and found evidence against spectral signatures of $\mathrm{H}_{2} \mathrm{O}$ tha tis theoretically expected in a clear atmosphere. All these studies have in common that they disagree with theoretical cloudfree atmosphere models, but could be much better explained by an opaque layer in the atmosphere either blocking only the pressure-broadened wings or the entire absorption feature when observed in low spectral resolution. Our measurements for HATP-19b agree well with these results and point toward the final statement of Fortney (2005), who reported that owing to the slanted viewing geometry of transmission spectroscopy, the detection of clear atmosphere will be rare. The only object for which sodium wings, which indicate a clear atmosphere, have been detected so far is the somewhat hotter HD 209458b (Sing et al. 2008a). But also in the temperature regime of $2000 \mathrm{~K}$ and above, it seems to be very common to find a spectrum more depleted in features than expected by clear atmosphere models, see the review by Madhusudhan et al. (2014).

Future follow-up observations of HAT-P-19b with HST/WFC3 from 1.1 to $1.7 \mu \mathrm{m}$ could strengthen our present indication for an atmosphere containing clouds or hazes by investigating the water absorption band at $1.4 \mu \mathrm{m}$.

\subsection{Long-term photometric variability}

H11 gathered photometry data with the HATnet telescopes from September 2007 to February 2008, high-resolution spectra for RV follow-up have been taken in August 2009 to August 2010. 
The authors did not mention any indication for stellar activity from photometry or spectroscopy. The RV jitter is low, $6.7 \mathrm{~m} \mathrm{~s}^{-1}$, and might be caused by instrumental systematics. However, the periodic photometric variations found by us in data taken from December 2011 to Octobre 2012 were interpreted as starspots moving in and out of view because of the larger amplitude of the variation in $B$ than in $V$. The same period of $\sim 37$ days detected in the HATnet data and STELLA data, consequently interpreted as stellar rotation period, shows an increase in amplitude from 2007 to 2012 by about a factor of 5 . Possible interpretations for this increase are a very homogeneous spot distribution in 2007 and a very inhomogeneous distribution in 2012. Another explanation is a change in activity level comparable with changes in the activity level during the 11-year solar cycle. Such stellar activity cycles have been detected in a variety of stars, see Strassmeier (2009) and references therein.

Knowing the rotation period of the star and its effective temperature, we have the opportunity to determine the age of HATP-19 using gyrochronology. Here, we assumed that the host star was not spun up by tidal interactions with its close-in planet. According to Eq. (15) in Husnoo et al. (2012), the timescale for synchronization of the rotation is above the age of the Universe and several orders of magnitudes higher than for systems with detected excess rotation that might be caused by tidal effects (Pont 2009; Husnoo et al. 2012; Poppenhaeger \& Wolk 2014). To apply the age determination from Barnes (2010), the effective temperature was converted empirically to a $B-V$ color by using the formula in Reed (1998). We derived $B-V=0.927 \pm 0.042$ from $T_{\text {eff }}=4990 \pm 130 \mathrm{~K}$. The errors arising from the unknown initial rotation period at zero age of a star between $0.12 \mathrm{~d}$ and $3.4 \mathrm{~d}$ result in an uncertainty of $0.2 \mathrm{Gyr}$ for that specific spectral type and rotation period. Taking all uncertainties into account (error in effective temperature, error in period determination, error in age determination), we estimate the age of the exoplanet host HAT-P-19 to be $5.5_{-1.3}^{+1.8}$ Gyr. This result refines the age estimation from $\mathrm{H} 11$ of $8.8 \pm 5.2 \mathrm{Gyr}$ based on stellar evolutionary tracks.

Knowledge of the rotation period of the star and its spectroscopic line broadening $v \sin i$ in principle allows determining the inclination of the star in the observer's line of sight (Walkowicz \& Basri 2013). It does not provide an absolute angle between the orbital plane of the planet and its host star rotation axis, but as we know the inclination of the planetary orbital plane from the transit measurement to be close to $90^{\circ}$, any significant deviation of the stellar inclination from the same value indicates a misalignment of the planetary system. However, for very slowly rotating stars like HAT-P-19, no meaningful conclusions can be drawn. The $v \sin i$ value of $0.7 \pm 0.5 \mathrm{~km} \mathrm{~s}^{-1}$ (taken from H11) in combination with our stellar rotation period of $\sim 35$ days allows for inclination angles from 10 to 90 degrees.

\section{Conclusion}

We reported GTC/OSIRIS spectroscopic observations of one transit of HAT-P-19b covering the wavelength range from 5600 to $7600 \AA$. The data allowed us to refine the transit parameters and the planetary orbital ephemeris. We derived an optical transmission spectrum of the Saturn-mass highly inflated exoplanet including the wavelength range of the $\mathrm{Na} \mathrm{D}$ line. We extracted a transmission spectrum using $200 \AA$ wide wavelength channels (spectral resolution $R \sim 30$ ) and another using $50 \AA$ wide channels $(R \sim 120)$. The result is in both cases a flat spectrum without additional absorption at any specific wavelength and without significant trends. No sodium absorption feature of the planetary atmosphere was detected.

We performed a monitoring campaign to search for a photometric variability of HAT-P-19 using the robotic $1.2 \mathrm{~m}$ telescope STELLA in the years 2011 and 2012. We found a clear periodic variation with $4.7 \pm 0.5 \mathrm{mmag}$ and $3.0 \pm 0.4 \mathrm{mmag}$ amplitude in the filters $V$ and $I$ and a period of $\sim 37$ days. The same periodicity of lower amplitude was found in the HATnet discovery photometry of 2007 and interpreted by us as the stellar rotation period. Our monitoring data covered the date of the spectroscopic transit observation and could therefore be used to correct the transit parameters for systematics caused by spots on the visible hemisphere. However, the influence of the spots on the transmission spectrum was found to be negligible. The obtained rotation period of the host star enabled us to refine the age estimate of the star by gyrochronology.

The derived transmission spectrum of HAT-P-19b favors a featureless gray atmosphere, but comparisons with theoretical models tuned to the HAT-P-19 system parameters showed that the achieved accuracy is not sufficient to place further constraints on the atmospheric composition. A model showing a Rayleighscattering slope and a solar-composition cloud-free atmosphere model with a pressure-broadened sodium line roughly agrees with the data. A transmission spectrum of higher $\mathrm{S} / \mathrm{N}$ can be obtained easily by follow-up observations of the same kind as presented here, since the current work is based on a singletransit observation in imperfect observing conditions. The lack of pre-ingress baseline made our modeling of underlying systematics in the light curve (detrending) more error prone and hence increased our final error bars in the transmission spectrum. If follow-up observations confirm the flat transmission spectrum to higher significance, the spectrum of HAT-P-19b would share an important property with the spectra of other close-in gas giants of about $1000 \mathrm{~K}$ atmospheric temperature investigated so far (Gibson et al. 2013a; Nikolov et al. 2014; Line et al. 2013): None of their transmission spectra can be explained by a cloud- or haze-free atmosphere model, they all show indications of an additional opacity source blocking parts of the probable atmosphere.

Acknowledgements. We thank the entire GTC staff for their help in executing this program. We thank Jonathan Fortney for providing the cloud-free solar composition atmospheric model for HAT-P-19b. We thank Joel Hartman for providing the HATnet photometry data. I.R. and E.H. acknowledge financial support from the Spanish Ministry of Economy and Competitiveness (MINECO) and the "Fondo Europeo de Desasarrollo Regional" (FEDER) through grant AYA201239612-C03-01. This work made use of PyAstronomy.

\section{References}

Aigrain, S., Pont, F., \& Zucker, S. 2012, MNRAS, 419, 3147

Bakos, G. Á., Noyes, R. W., Kovács, G., et al. 2007, ApJ, 656, 552

Barnes, S. A. 2010, ApJ, 722, 222

Bean, J. L., Miller-Ricci Kempton, E., \& Homeier, D. 2010, Nature, 468, 669 Berdyugina, S. V. 2005, Liv. Rev. Sol. Phys., 2, 8

Berta, Z. K., Charbonneau, D., Bean, J., et al. 2011, ApJ, 736, 12

Bertin, E., \& Arnouts, S. 1996, A\&AS, 117, 393

Broeg, C., Fernández, M., \& Neuhäuser, R. 2005, Astron. Nachr., 326, 134

Brown, T. M. 2001, ApJ, 553, 1006

Bundy, K. A., \& Marcy, G. W. 2000, PASP, 112, 1421

Charbonneau, D., Brown, T. M., Noyes, R. W., \& Gilliland, R. L. 2002, ApJ, 568,377

Claret, A. 2000, A\&A, 363, 1081

Claret, A. 2004, A\&A, 428, 1001

Claret, A., Hauschildt, P. H., \& Witte, S. 2013, A\&A, 552, A16

Colón, K. D., Ford, E. B., Redfield, S., et al. 2012, MNRAS, 419, 2233

Czesla, S., Huber, K. F., Wolter, U., Schröter, S., \& Schmitt, J. H. M. M. 2009, A\&A, 505, 1277 
Désert, J.-M., Vidal-Madjar, A., Lecavelier Des Etangs, A., et al. 2008, A\&A, 492, 585

Désert, J.-M., Sing, D., Vidal-Madjar, A., et al. 2011, A\&A, 526, A12

Eastman, J., Siverd, R., \& Gaudi, B. S. 2010, PASP, 122, 935

Fortney, J. J. 2005, MNRAS, 364, 649

Fortney, J. J., Sudarsky, D., Hubeny, I., et al. 2003, ApJ, 589, 615

Fortney, J. J., Saumon, D., Marley, M. S., Lodders, K., \& Freedman, R. S. 2006, ApJ, 642, 495

Fortney, J. J., Shabram, M., Showman, A. P., et al. 2010, ApJ, 709, 1396

Gibson, N. P., Aigrain, S., Barstow, J. K., et al. 2013a, MNRAS, 428, 3680

Gibson, N. P., Aigrain, S., Barstow, J. K., et al. 2013b, MNRAS, 436, 2974

Gillon, M., Pont, F., Moutou, C., et al. 2006, A\&A, 459, 249

Hartman, J. D., Bakos, G. Á., Torres, G., et al. 2009, ApJ, 706, 785

Hartman, J. D., Bakos, G. Á., Sato, B., et al. 2011, ApJ, 726, 52

Hellier, C., Anderson, D. R., Collier Cameron, A., et al. 2010, ApJ, 723, L60

Helling, C., Woitke, P., \& Thi, W.-F. 2008, A\&A, 485, 547

Horne, K. 1986, PASP, 98, 609

Hubbard, W. B., Fortney, J. J., Lunine, J. I., et al. 2001, ApJ, 560, 413

Hubeny, I., Burrows, A., \& Sudarsky, D. 2003, ApJ, 594, 1011

Huitson, C. M., Sing, D. K., Pont, F., et al. 2013, MNRAS, 434, 3252

Husnoo, N., Pont, F., Mazeh, T., et al. 2012, MNRAS, 422, 3151

Jenkins, J. M., Caldwell, D. A., \& Borucki, W. J. 2002, ApJ, 564, 495

Johnson, J. A., Winn, J. N., Narita, N., et al. 2008, ApJ, 686, 649

Jordán, A., Espinoza, N., Rabus, M., et al. 2013, ApJ, 778, 184

Lecavelier Des Etangs, A., Pont, F., Vidal-Madjar, A., \& Sing, D. 2008a, A\&A, 481, L83

Lecavelier Des Etangs, A., Vidal-Madjar, A., Désert, J.-M., \& Sing, D. 2008b, A\&A, 485, 865

Line, M. R., Knutson, H., Deming, D., Wilkins, A., \& Desert, J.-M. 2013, ApJ, 778,183

Lodders, K. 1999, ApJ, 519, 793

Madhusudhan, N., Knutson, H., Fortney, J. J., \& Barman, T. 2014, Protostars and Planets VI, 914, 739

Mandel, K., \& Agol, E. 2002, ApJ, 580, L171

Moutou, C., Coustenis, A., Schneider, J., et al. 2001, A\&A, 371, 260

Murgas, F., Pallé, E., Zapatero Osorio, M. R., et al. 2014, A\&A, 563, A41
Nikolov, N., Sing, D. K., Pont, F., et al. 2014, MNRAS, 437, 46 Nikolov, N., Sing, D. K., Burrows, A. S., et al. 2015, MNRAS, 447, 463 Pont, F. 2009, MNRAS, 396, 1789

Pont, F., Knutson, H., Gilliland, R. L., Moutou, C., \& Charbonneau, D. 2008 , MNRAS, 385, 109

Pont, F., Sing, D. K., Gibson, N. P., et al. 2013, MNRAS, 432, 2917

Poppenhaeger, K., \& Wolk, S. J. 2014, A\&A, 565, L1

Rauer, H., Collier Cameron, A., Barnes, J., \& Harris, A. 2000, BAAS, 32, 1053

Reed, B. C. 1998, J. Roy. Astron. Soc. Canada, 92, 36

Schwarz, G. 1978, Ann. Statist., 6, 461

Seager, S., \& Sasselov, D. D. 2000, ApJ, 537, 916

Sing, D. K., Vidal-Madjar, A., Désert, J.-M., Lecavelier des Etangs, A., \& Ballester, G. 2008a, ApJ, 686, 658

Sing, D. K., Vidal-Madjar, A., Lecavelier des Etangs, A., et al. 2008b, ApJ, 686, 667

Sing, D. K., Désert, J.-M., Fortney, J. J., et al. 2011a, A\&A, 527, A73

Sing, D. K., Pont, F., Aigrain, S., et al. 2011b, MNRAS, 416, 1443

Sing, D. K., Huitson, C. M., Lopez-Morales, M., et al. 2012, MNRAS, 426, 1663

Sing, D. K., Lecavelier des Etangs, A., Fortney, J. J., et al. 2013, MNRAS, 436, 2956

Sing, D. K., Wakeford, H. R., Showman, A. P., et al. 2015, MNRAS, 446, 2428 Southworth, J. 2008, MNRAS, 386, 1644

Southworth, J., Maxted, P. F. L., \& Smalley, B. 2004, MNRAS, 349, 547

Southworth, J., Smalley, B., Maxted, P. F. L., Claret, A., \& Etzel, P. B. 2005, MNRAS, 363, 529

Southworth, J., Bruntt, H., \& Buzasi, D. L. 2007a, A\&A, 467, 1215

Southworth, J., Wheatley, P. J., \& Sams, G. 2007b, MNRAS, 379, L11

Stevenson, K. B., Bean, J. L., Seifahrt, A., et al. 2014, AJ, 147, 161

Strassmeier, K. G. 2009, A\&ARv, 17, 251

Strassmeier, K. G., Granzer, T., Weber, M., et al. 2004, Astron. Nachr., 325, 527

Tregloan-Reed, J., Southworth, J., \& Tappert, C. 2013, MNRAS, 428, 3671 Walkowicz, L. M., \& Basri, G. S. 2013, MNRAS [arXiv: 1309.2159]

Weber, M., Granzer, T., \& Strassmeier, K. G. 2012, in SPIE Conf. Ser., 8451

Winn, J. N., Holman, M. J., Torres, G., et al. 2008, ApJ, 683, 1076

Woitke, P., \& Helling, C. 2003, A\&A, 399, 297 
M. Mallonn et al.: Transmission spectroscopy of HAT-P-19b

Table 1. Dependence of the derived transit parameters on the model function used for detrending.

\begin{tabular}{ccccccc}
\hline \hline $\begin{array}{c}\text { Model } \\
\text { function }\end{array}$ & BIC & $\begin{array}{c}\mathrm{rms} \\
(\mathrm{mmag})\end{array}$ & $\beta$ & $k=R_{b} / R_{\mathrm{A}}$ & $a / R_{\mathrm{A}}$ & $\begin{array}{c}i \\
(\mathrm{deg})\end{array}$ \\
\hline$p\left(z^{4}\right)$ & 292.1 & 0.42 & 1.08 & $0.1406 \pm 0.0012$ & $12.32 \pm 0.21$ & $89.20 \pm 0.31$ \\
$\boldsymbol{p}\left(z^{3}\right)$ & $\mathbf{2 7 9 . 8}$ & $\mathbf{0 . 4 3}$ & $\mathbf{1 . 1 2}$ & $\mathbf{0 . 1 3 9 0} \pm \mathbf{0 . 0 0 1 2}$ & $\mathbf{1 2 . 3 7} \pm \mathbf{0 . 2 1}$ & $\mathbf{8 8 . 8 9} \pm \mathbf{0 . 3 2}$ \\
$p\left(z^{2}\right)$ & 328.7 & 0.48 & 1.58 & $0.1341 \pm 0.0020$ & $12.41 \pm 0.43$ & $88.95 \pm 0.61$ \\
$p\left(z^{3}\right)+p\left(x^{1}\right)$ & 292.2 & 0.43 & 1.17 & $0.1379 \pm 0.0012$ & $12.46 \pm 0.25$ & $89.12 \pm 0.45$ \\
$p\left(z^{3}\right)+p\left(y^{1}\right)$ & 294.4 & 0.43 & 1.21 & $0.1382 \pm 0.0013$ & $12.23 \pm 0.29$ & $88.95 \pm 0.43$ \\
$p\left(z^{2}\right)+p\left(t^{2}\right)$ & 339.7 & 0.48 & 1.56 & $0.1343 \pm 0.0015$ & $12.41 \pm 0.33$ & $89.20 \pm 0.56$ \\
$p\left(t^{4}\right)$ & 304.3 & 0.45 & 1.09 & $0.1399 \pm 0.0014$ & $12.24 \pm 0.27$ & $88.97 \pm 0.46$ \\
$p\left(t^{3}\right)$ & 319.1 & 0.48 & 1.36 & $0.1335 \pm 0.0018$ & $12.56 \pm 0.44$ & $89.19 \pm 0.64$ \\
$p\left(t^{2}\right)$ & 316.9 & 0.50 & 1.51 & $0.1354 \pm 0.0021$ & $12.51 \pm 0.36$ & $88.85 \pm 0.59$ \\
$p\left(F W H M^{4}\right)$ & 337.4 & 0.47 & 1.71 & $0.1341 \pm 0.0019$ & $12.46 \pm 0.43$ & $88.61 \pm 0.78$ \\
$p\left(F W H M^{3}\right)$ & 393.0 & 0.54 & 1.99 & $0.1329 \pm 0.0027$ & $12.38 \pm 0.51$ & $88.95 \pm 0.95$ \\
\hline
\end{tabular}

Notes. The notation $p\left(j^{i}\right)$ refers to a polynomial of degree $i$ of parameter $j$, e.g., $p\left(z^{3}\right)$ denotes a polynomial of third degree with respect to airmass $z$. The model function used for the final light curve analysis is printed in boldface. 
Table 3. Characteristics of the extracted transit light-curves.

\begin{tabular}{|c|c|c|c|c|c|}
\hline $\begin{array}{c}\text { Wavelength } \\
(\AA)\end{array}$ & $\beta$ & $\begin{array}{c}\mathrm{rms} \\
(\mathrm{mmag})\end{array}$ & $\begin{array}{c}u_{\mathrm{A}} \\
\text { fitted }\end{array}$ & $\begin{array}{c}v_{\mathrm{A}} \\
\text { calculated } \\
\end{array}$ & $k=r_{b} / r_{A}$ \\
\hline $5617-7616$ & 1.12 & 0.43 & 0.5736 & 0.1376 & $0.1390 \pm 0.0012$ \\
\hline $5617-5816$ & 1.16 & 0.73 & 0.6087 & 0.0988 & $0.1412 \pm 0.0013$ \\
\hline $5817-6016$ & 1.11 & 0.69 & 0.6127 & 0.1132 & $0.1382 \pm 0.0015$ \\
\hline $6017-6216$ & 1.27 & 0.62 & 0.6233 & 0.1230 & $0.1390 \pm 0.0014$ \\
\hline $6217-6416$ & 1.04 & 0.68 & 0.5964 & 0.1258 & $0.1392 \pm 0.0014$ \\
\hline $6417-6616$ & 1.02 & 0.71 & 0.5663 & 0.1450 & $0.1392 \pm 0.0014$ \\
\hline $6617-6816$ & 1.15 & 0.64 & 0.5708 & 0.1442 & $0.1383 \pm 0.0012$ \\
\hline $6817-7016$ & 1.19 & 0.68 & 0.5726 & 0.1495 & $0.1388 \pm 0.0010$ \\
\hline $7017-7216$ & 1.00 & 0.66 & 0.5586 & 0.1443 & $0.1392 \pm 0.0012$ \\
\hline $7217-7416$ & 1.00 & 0.58 & 0.5155 & 0.1529 & $0.1407 \pm 0.0011$ \\
\hline $7417-7616$ & 1.06 & 0.59 & 0.5010 & 0.1570 & $0.1412 \pm 0.0012$ \\
\hline $5617-5666$ & 1.33 & 1.21 & 0.6021 & 0.0989 & $0.1422 \pm 0.0027$ \\
\hline $5667-5716$ & 1.05 & 1.10 & 0.5722 & 0.0969 & $0.1423 \pm 0.0023$ \\
\hline $5717-5766$ & 1.27 & 1.09 & 0.5748 & 0.0921 & $0.1408 \pm 0.0021$ \\
\hline $5767-5816$ & 1.02 & 0.99 & 0.6060 & 0.1068 & $0.1380 \pm 0.0017$ \\
\hline $5817-5866$ & 1.00 & 0.94 & 0.6293 & 0.1204 & $0.1358 \pm 0.0017$ \\
\hline $5867-5916$ & 1.08 & 1.02 & 0.6034 & 0.0930 & $0.1394 \pm 0.0018$ \\
\hline $5917-5966$ & 1.00 & 1.06 & 0.6470 & 0.1118 & $0.1359 \pm 0.0017$ \\
\hline $5967-6016$ & 1.03 & 1.03 & 0.5886 & 0.1246 & $0.1379 \pm 0.0019$ \\
\hline $6017-6066$ & 1.11 & 1.04 & 0.6163 & 0.1278 & $0.1377 \pm 0.0026$ \\
\hline $6067-6116$ & 1.22 & 1.09 & 0.6390 & 0.1158 & $0.1345 \pm 0.0020$ \\
\hline $6117-6166$ & 1.20 & 1.21 & 0.6026 & 0.1221 & $0.1414 \pm 0.0022$ \\
\hline $6167-6216$ & 1.01 & 1.03 & 0.5902 & 0.1263 & $0.1389 \pm 0.0017$ \\
\hline $6217-6266$ & 1.00 & 0.91 & 0.5626 & 0.1246 & $0.1397 \pm 0.0015$ \\
\hline $6267-6316$ & 1.18 & 0.95 & 0.5931 & 0.1248 & $0.1384 \pm 0.0016$ \\
\hline $6317-6366$ & 1.13 & 1.05 & 0.5843 & 0.1307 & $0.1378 \pm 0.0025$ \\
\hline $6367-6416$ & 1.00 & 0.99 & 0.5779 & 0.1232 & $0.1393 \pm 0.0015$ \\
\hline $6417-6466$ & 1.04 & 0.90 & 0.5620 & 0.1225 & $0.1401 \pm 0.0018$ \\
\hline $6467-6516$ & 1.04 & 0.93 & 0.5867 & 0.1352 & $0.1381 \pm 0.0019$ \\
\hline $6517-6566$ & 1.41 & 0.92 & 0.5425 & 0.1703 & $0.1402 \pm 0.0016$ \\
\hline $6567-6616$ & 1.08 & 1.06 & 0.5467 & 0.1510 & $0.1379 \pm 0.0021$ \\
\hline $6617-6666$ & 1.26 & 1.02 & 0.5639 & 0.1415 & $0.1391 \pm 0.0018$ \\
\hline $6667-6716$ & 1.40 & 0.95 & 0.5864 & 0.1419 & $0.1364 \pm 0.0016$ \\
\hline $6717-6766$ & 1.31 & 0.87 & 0.5655 & 0.1483 & $0.1386 \pm 0.0016$ \\
\hline $6767-6816$ & 1.06 & 0.82 & 0.5979 & 0.1450 & $0.1383 \pm 0.0015$ \\
\hline $6817-6866$ & 1.00 & 0.93 & 0.5371 & 0.1472 & $0.1394 \pm 0.0018$ \\
\hline $6867-6916$ & 1.43 & 0.95 & 0.5530 & 0.1533 & $0.1390 \pm 0.0014$ \\
\hline $6917-6966$ & 1.00 & 1.05 & 0.6084 & 0.1484 & $0.1375 \pm 0.0017$ \\
\hline $6967-7016$ & 1.15 & 1.00 & 0.5354 & 0.1492 & $0.1391 \pm 0.0017$ \\
\hline $7017-7066$ & 1.05 & 1.36 & 0.4911 & 0.1421 & $0.1369 \pm 0.0029$ \\
\hline $7067-7116$ & 1.01 & 0.95 & 0.5573 & 0.1498 & $0.1392 \pm 0.0014$ \\
\hline $7117-7166$ & 1.05 & 1.13 & 0.5190 & 0.1434 & $0.1393 \pm 0.0024$ \\
\hline $7167-7216$ & 1.02 & 1.39 & 0.5635 & 0.1414 & $0.1385 \pm 0.0022$ \\
\hline $7217-7266$ & 1.01 & 0.97 & 0.5161 & 0.1494 & $0.1404 \pm 0.0014$ \\
\hline $7267-7316$ & 1.00 & 0.82 & 0.4732 & 0.1568 & $0.1418 \pm 0.0016$ \\
\hline $7317-7366$ & 1.00 & 0.93 & 0.4958 & 0.1527 & $0.1403 \pm 0.0016$ \\
\hline $7367-7416$ & 1.04 & 0.96 & 0.5532 & 0.1530 & $0.1382 \pm 0.0016$ \\
\hline $7417-7466$ & 1.00 & 0.92 & 0.4955 & 0.1578 & $0.1406 \pm 0.0017$ \\
\hline $7467-7516$ & 1.07 & 0.81 & 0.5077 & 0.1572 & $0.1399 \pm 0.0015$ \\
\hline $7517-7566$ & 1.00 & 0.87 & 0.4793 & 0.1602 & $0.1394 \pm 0.0015$ \\
\hline $7567-7616$ & 1.00 & 0.98 & 0.5008 & 0.1510 & $0.1432 \pm 0.0018$ \\
\hline $7617-7666$ & 1.13 & 1.03 & 0.5131 & 0.1543 & $0.1394 \pm 0.0018$ \\
\hline $5667-5866$ & 1.13 & 0.65 & 0.6002 & 0.1046 & $0.1395 \pm 0.0013$ \\
\hline $5867-5916$ & 1.08 & 1.02 & 0.6034 & 0.0930 & $0.1394 \pm 0.0018$ \\
\hline $5917-6516$ & 1.25 & 0.56 & 0.5908 & 0.1243 & $0.1386 \pm 0.0012$ \\
\hline $6537-6587$ & 1.31 & 1.05 & 0.5449 & 0.1727 & $0.1397 \pm 0.0020$ \\
\hline $6587-7666$ & 1.14 & 0.46 & 0.5296 & 0.1492 & $0.1391 \pm 0.0011$ \\
\hline
\end{tabular}

